\title{
Marker-less Tracking for Respiratory Motion Correction in Nuclear Medicine
}

\author{
Majdi R. Alnowam, E. Lewis, M. Guy and K. Wells
}

\begin{abstract}
AbstractThis paper present preliminary work in developing a method of using a marker-less tracking system to analyze the natural temporal variations in chest wall configuration during breathing, thus avoiding reliance on a limited number of fiducial markers. This involves using a marker-less video capture of the motion of the abdominal-chest surface and the development of a B-spline model to parameterize this motion. The advantage of the marker-less system that is noninvasive and non-ionizing, thus facilitating high throughput without the need for marker-based patient set-up time
\end{abstract}

\section{INTRODUCTION}

One of the current major challenges in nuclear emission tomography is respiratory motion correction. Respiratory motion during the emission data acquisition process leads to blurred images, thus challenging diagnosis, planning and follow-up processes. There is significant clinical evidence on the effect that respiratory motion has on lesions localization [1], [2]. Correspondingly, significant tumor motion and variation in lung volume has been reported in [3] in which images reconstructed without respiratory motion compensation were studied. A significant research focus is in developing new approaches to monitor and track respiratory motion during data acquisition[4]. This may involve techniques to correct motion during reconstruction e.g. Reyes et al. [3] or using a marker-based tracking system to monitor the body motion during data acquisition, e.g. Nehmeh et al.[5] and Wolthaus et al.,[6]. However, using a marker-based approach to track the respiratory motion is undesirable in a clinical environment as this may limit throughput and has also also often involve making gross assumptions about volumetric internal motion from a limited set of fiducial elements.

This paper present preliminary work in developing a method of using a marker-less system to analyze the natural temporal variations in chest wall configuration during breathing, without relying on finite number of markers. Initial pilot study was undertaken using a set of volunteers, imaged using the 3D-MD video capture system (marker-less). A markerless system is highly attractive in terms patient throughput.

Majdi R. Alnowam, E. Lewis, and K. Wells are from the Centre for Vision, Speech and Signal Processing, FEPS, University of Surrey, Guildford, Surrey GU2 7XH, UK.

M. Guy is from Medway Maritime Hospital, Windmill Road, Gillingham, Kent, ME7 5NY, UK.

Further author information E-mail: m.alnowami@surrey.ac.uk.
The method involve creating a patient-specific model for the anterior surface using a B-spline model for each phase or frame within the respiratory cycle and then detects the perturbation of the pattern as motion. This approach is a part of a larger scheme [7], [8] to achieve motion correction in nuclear medicine.

\section{Methodology}

One of the main constraints in using a 3D stereo camera system without markers is establishing inter-frame pointcorrespondence. Unlike prior work on facial landmark tracking [9] where landmarks can be relatively easily determined, segmentation or identification of a set of reference points on the anterior surface is challenging since the anterior surface is primarily smooth (with insignificant landmarks). Moreover, a marker-less system is highly attractive in terms of patient throughput but marker-less commercial solutions tend to generate Gigabytes of data per second of capture, which is cumbersome, at least, at the initial development stage and impractical for clinical or routine development work, with scanning times of potentially up to 30 minutes. In the method proposed here, a two dimensional base grid was defined in the $\left(x_{i}, y_{i}\right)$ plane parallel to anterior surface and in the ground level as illustrated in Figure 4, where $\mathrm{i}$ is the number of nodes. Then, from each node $\left(x_{i}, y_{i}\right)$, perpendicular lines were projected towards a 3D points cloud of the surface generated by the marker-less tracking system. The resulting intersection points between the projected lines and the 3D cloud will define a set of control points CP. Thus , by repeating this process for each frame generated by the tracking system, $\mathrm{N}$ set of control points will be generated $C P_{n}$ where $\mathrm{n}$ is the number of frame generated by the marker-less system. These control points $C P_{N}$ will be used as the B-spline model parameters. The B spline model serves three main purposes: (i) it reduces the size of the dataset needed to adequately describe a particular observed state of the anterior surface; (ii) it regulates the number of data points used so that comparing observation and prediction can be easily achieved;(iii) the resulting points describing a B-splines surface model for each respiratory phase can be used as input points for subsequent analysis.

\section{A. Materials:}

An initial pilot study was undertaken using a set of volunteers, imaged using the 3D-MD video capture tracking 
system. Adding the dimension of time to high-precision 3D well enable a deeper understanding of true temporal motion of the anterior surface during respiration. The video capture tracking system comprises six synchronized digital video cameras and a standard continuous IR lighting system. The cameras are arranged into two banks of three cameras, with each bank comprising two monochrome cameras (top and bottom) and one color camera (middle). The two banks of cameras are arranged to capture the left and right sides of the anterior surface. This system projects an infra-red speckle pattern onto a surface, then detects the perturbation of the pattern as motion. The system has a geometric accuracy of less than $0.2 \mathrm{~mm}$ RMS with 0.5 milliseconds capture speed generating data at a fixed rate of $60 \mathrm{frame} / \mathrm{sec}$.

\section{B. Volunteers Preparation:}

In this initial study a set of seven male and five female volunteers having a significant variation in body shape, with body mass indices (BMI) ranging between $27.8 \mathrm{~kg} / \mathrm{m}^{2}$ and $21.3 \mathrm{~kg} / \mathrm{m}^{2}$, were imaged. Volunteers were asked to maintain the same overall position during the acquisitions and to breathe quietly for 10s. No others particular instruction regarding the breathing pattern was given. All volunteers were imaged in the supine position. Generally, All volunteer were undress from the waist up. The reason for this was to avoid any wrinkles that could result from the clothes during imaging.

\section{Marker-less Motion Capture Data:}

Figure 1(a) shows an example set of surfaces created by the 3D-MD video capture system for one of the female volunteers. Each layer represents a surface of the volunteer's thoracic and abdomen at a different phases/states during the breathing cycle. The Cartesian coordinate data can be interrogated at different time points up to a maximum of 60 frames per second Figure 1(b). The size of a raw data generated by the 3DMD marker-less system for one frame at time $\mathrm{t}$ is more than $45 \mathrm{kB}$. Thus for 60 frames per second of capture there will be $38 \mathrm{~GB}$ of data generated per second, which is impractical for clinical or routine development work, with scanning times of potentially up to 30 minutes.

\section{B-spline Model}

As mentioned in the previous section and shown in Figure 1(b), the cloud points created from the 3D camera is not correlated. Therefore, to build an inter-frame to frame relationship you need to synchronized the cloud points configuration. In the method proposed here, a two dimensional base grid was defined in the $\left(x_{i}, y_{i}\right)$ plane parallel to anterior surface and in the ground level as illustrated in Figure 2, where $\mathrm{i}$ is the number of node. Then, from each node $\left(x_{i}, y_{i}\right)$, a perpendicular lines were projected towards a 3D points cloud of the surface generated by the marker-less tracking system.

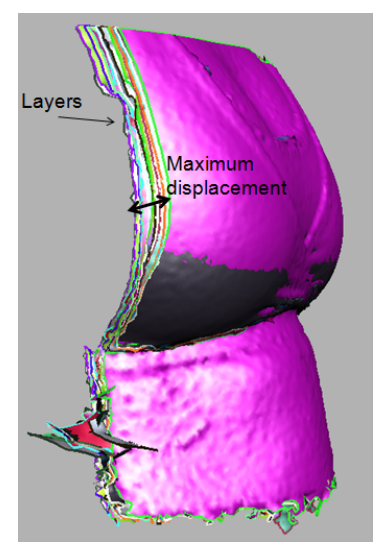

(a) Exemplar set of surface renders of female subject at different phases of respiration

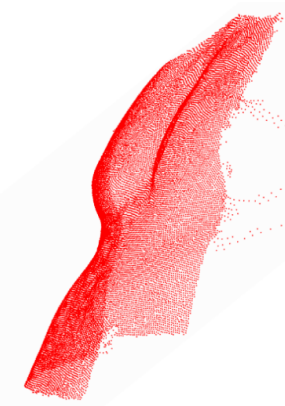

(b) The Cartesian coordinate data for surface renders for male subject at one phases of respiration.
Fig. 1. An example set of surfaces created by the 3D-MD video capture system.

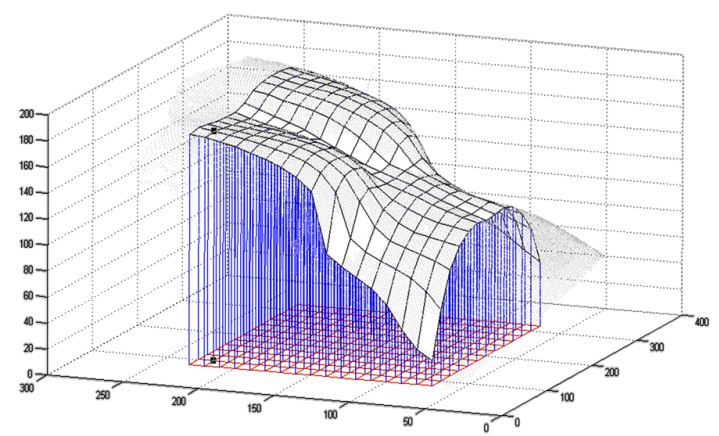

Fig. 2. A two dimensional base grid was defined in the $\left(x_{i}, y_{i}\right)$ plane parallel to anterior surface and in the ground level, where $i$ is the number of node. Then, from each node (xi,yi), a perpendicular lines were projected towards a $3 \mathrm{D}$ points cloud of the surface generated by the marker-less tracking system.

The Intersection points between the perpendicular line and the $3 \mathrm{D}$ cloud generate the $\mathrm{B}$-spline Control points $C P$.

In general, a B-spline surface $Q$ of order $k$ in the $u$ direction and order $l$ in the $v$ direction is a bivariate vectorvalued piecewise function of the form:

$$
Q(u, v)=\sum_{i=1}^{n+1} \sum_{j=1}^{m+1} C P_{i, j} N_{i, k}(u) M_{j, l}(v),
$$

where $C P_{i, j} \mathrm{~s}$ are the vertices (control points) of a polygonal control net.These are a set of $m+1$ rows and $n+1$ columns $C P_{i, j}$, where $0 \leq i \leq m$ and $0 \leq j \leq n$ ( see figure 3).

Furthermore, $N_{i, k}(u)$ and $M_{j, l}(v)$ are the B-spline basis function in the bi-parametric $u$ and $v$ directions, respectively. The $i-t h \mathrm{~B}$-spline basis function of degree $k$, written as $N_{i, k}(u)$, as well as the $j-t h \mathrm{~B}$-spline basis function of degree $l$, written as $M_{j, l}(v)$ are defined recursively as follows:

$$
N_{i, k}(u)=\frac{\left(N_{i, k-1}(u)\left(u-x_{i}\right)\right)}{\left(x_{i+k-1}-x_{i}\right)}+\frac{\left(N_{i+1, k-1}(u)\left(x_{i+k}-u\right)\right)}{\left(x_{i+k}-x_{i+1}\right)}
$$




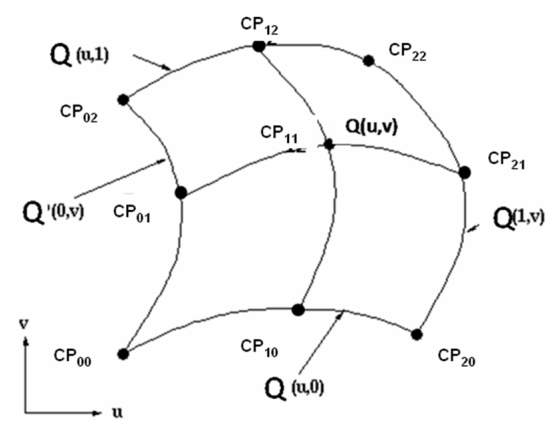

Fig. 3. Illustrates $C P_{i, j}$ s the vertices of a polygonal control net for Bspline surface $Q$ of order $k$ in the $u$ direction and order $l$ in the $v$ direction

$$
N_{i, 1}(u)=\left\{\begin{array}{rr}
1 & : x_{i} \leq u \leq x_{i+1} \\
0 & : \text { otherwise }
\end{array}\right.
$$

and

$$
\begin{gathered}
M_{j, l}(v)=\frac{\left(M_{j, l-1}(v)\left(v-y_{i}\right)\right)}{\left(y_{j+l-1}-y_{j}\right)}+\frac{\left(M_{j+1, l-1}(v)\left(y_{j+l}-v\right)\right)}{\left(y_{j+l}-y_{j+1}\right)} \\
M_{j, 1}(v)=\left\{\begin{array}{cc}
1 & : y_{j} \leq v \leq y_{j+1} \\
0 & : \text { otherwise }
\end{array}\right.
\end{gathered}
$$

where $x_{i}$ and $y_{j}$ are elements of the knot vectors. The only requirements for the knot vectors is that they satisfy the relation $x_{i} \leq x_{i+1}$ and $y_{j} \leq y_{j+1}$; i.e., the vector is a monotonically increasing series of real numbers. In a uniform knot vector, individual knot values are evenly spaced. An open uniform knot vectors has a multiplicity of knot values at the end equal to the order $k$ of the B-spline basis function. Formally, an open uniform knot vector $x_{i}$ is given by:

$$
x_{i}=\left\{\begin{array}{lr}
0 & : 1 \leq i \leq k \\
i-k & : k+1 \leq i \leq n+1 \\
n-k+2 & : n+2 \leq i \leq n+k+1
\end{array}\right.
$$

and

$$
y_{i}=\left\{\begin{array}{lr}
0 & : 1 \leq j \leq l \\
j-l & : l+1 \leq j \leq m+1 \\
m-l+2 & : m+2 \leq j \leq m+l+1
\end{array}\right.
$$

The maximum possible order $(j, k)$ of the surface in each parametric direction $(u, v)$ is equal to the number of control polygon vertices in that direction $(n, m)$. The continuity of the surface in each parametric direction is two less than the order in each direction. The generated surface lies within the convex hull of the control net formed by the union of all the convex hulls of $k, l$, and neighbouring control net vertices.

\section{E. Result and analysis:}

The B-spline model can be summarized with reference to the Figure 4. Figure 4(A) shows an example set of surfaces created by the 3D-MD video capture system for one of the male volunteers. Each layer represents a surface of the volunteer's Thoracic and Abdomen at a different phases/frame $F_{n}$ during the breathing cycle.

The number of initial coordinates points generated by the 3DMD marker-less system for one frame at time $t$ is more

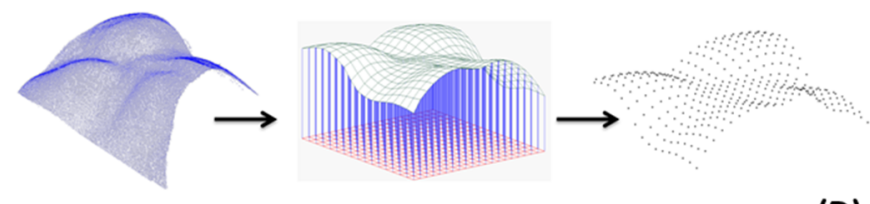

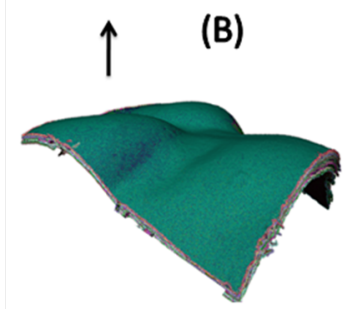

(A)
(C)

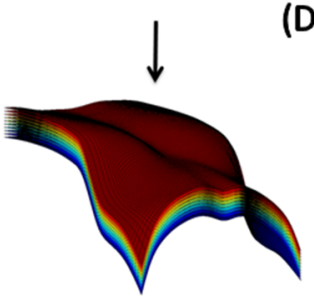

(E)
Fig. 4. Illustrates a method of transforming a large uncorrelated set of layers created by the video capture system into a correlated layers. (A) illustrates the data set generated to each subjects. (B) Indicates the cartesian coordinate for one layer within the respiration data. (C) A two dimensional base grid defined in the $(x, y)$ plane parallel to the anterior surface and in the ground level as illustrated. moreover, it illustrates the perpendicular lines projected towards the $3 \mathrm{~d}$ cloud (D) The control points of the B-spline model (E) illustrates all the data frames or phases

than $45 \mathrm{kB}$. Thus for 60 frames per second of capture there will be $2.7 \mathrm{MB}$ points generated per second. This represent $38 \mathrm{~GB}$ of data generated per second, which is impractical for clinical or routine development work, with scanning times of potentially up to 30 minutes. Moreover, the resulting meshing algorithms do not provide inter-frame point correspondence, thus obfuscating tracking the displacement of point across a set of frames. In this initial representation, $13 * 17$ sized array was arbitrarily selected, generating a set of 221 uniformly distributed control points that were linked to form a mesh over the anterior surface. The size of the control points array was determined empirically after some initial experimentation with the data. B-splines are then fitted to the high density coordinate data. Figure 4 (B) shows an example plot of the high density Cartesian data of a volunteer's anterior surface, obtained from the dynamic stereo camera; it also illustrates the mesh created to model this surface. Figure 4 (E) shows a set of interpolated frames from the resulting B spline model overlaid each other; this shows excellent visual agreement between the model and data. Table I illustrates the results obtained from scanning the aforementioned volunteer cohort. The average error associated with this model is $0.52 \pm 0.11 \mathrm{~mm}$. Figure 5 illustrated the error associated with each interpolated point.With 221 control points, and the storage of the B-spline parameters means that each frame now requires $6 \mathrm{kB}$ per frame so that for 60 frames per second there would be $\approx 0.4 \mathrm{MB}$ per second, representing a saving of more than $90 \%$ over storing the raw frame data. Methods to optimise the number of control points and the resulting fitting process are currently under development. In addition, investigations are underway to examine different forms of breathing and their effect on the parameters used to describe the surface in question. 


\begin{tabular}{|c|c|c|c|c|}
\hline $\begin{array}{l}\text { Volunteer } \\
\text { No. }\end{array}$ & Gender & $\begin{array}{l}\text { BMI } \\
\left(\mathrm{kg} / m^{2}\right)\end{array}$ & $\begin{array}{l}\text { TS Displace- } \\
\text { ment }(\mathrm{mm})\end{array}$ & $\begin{array}{l}\text { AS Displace- } \\
\text { ment }(\mathrm{mm})\end{array}$ \\
\hline 1 & $\mathrm{M}$ & 28.2 & 12.5 & 39.8 \\
\hline 2 & M & 24.3 & 11.3 & 27.9 \\
\hline 3 & M & 25.2 & 24.6 & 8.5 \\
\hline 6 & $\mathrm{M}$ & 21.8 & 18.4 & 11.0 \\
\hline 7 & $\mathrm{M}$ & 27.8 & 6.8 & 12.7 \\
\hline 4 & M & 24.8 & 10.3 & 10.8 \\
\hline 9 & M & 21.4 & 13.6 & 25.7 \\
\hline 5 & $\mathrm{~F}$ & 25.2 & 13.5 & 9.6 \\
\hline 8 & $\mathrm{~F}$ & 24.6 & 7.4 & 15.6 \\
\hline 10 & $\mathrm{~F}$ & 22.2 & 7.6 & 22.7 \\
\hline 11 & $\mathrm{~F}$ & 25.5 & 7.1 & 20.3 \\
\hline 12 & $\mathrm{~F}$ & 21.7 & 5.1 & 24.6 \\
\hline \multicolumn{2}{|c|}{ Cohort average } & 23.6 & 10.7 & 16.0 \\
\hline \multicolumn{2}{|c|}{ Standard Deviation } & 2.3 & 5.6 & 9.5 \\
\hline \multicolumn{2}{|c|}{ Max } & 28.2 & 24.6 & 39.8 \\
\hline \multicolumn{2}{|c|}{ Min } & 21.4 & 5.1 & 8.5 \\
\hline
\end{tabular}

TABLE I

MEAN EXPERIMENTAL SURFACE DISPLACEMENTS FOR BOTH CHEST AND ABDOMINAL REGION DURING A 10S BREATHING CYCLE ACQUISITION FOR A GROUP OF 12 MALE AND FEMALE VOLUNTEERS.
[3] M. Reyes, G. Malandain, P. M. Koulibaly, M. A. Gonzalez-Ballester, and J. Darcourt, "Model-based respiratory motion compensation for emission tomography image reconstruction," Physics in Medicine and Biology 52(12), pp. 3579-3600, 2007.

[4] A. Rahmim, O. Rousset, and H. Zaidi 2, pp. 251-266, Apr. 2007.

[5] S. A. Nehmeh, Y. E. Erdi, K. E. Rosenzweig, H. Schoder, S. M. Larson, O. D. Squire, and J. L. Humm J Nucl Med 44(10), pp. 1644-8, 2003.

[6] J. W. H. Wolthaus, M. V. Herk, S. H. Muller, J. S. A. Belderbos, J. V. Lebesque, J. A. D. Bois, M. M. G. Rossi, and E. M. F. Damen, "Fusion of respiration-correlated pet and ct scans: correlated lung tumour motion in anatomical and functional scans," Physics in Medicine and Biology 50, pp. 1569-1583, April 2005.

[7] K. Wells, B. Goswami, A. Rahni, J. Jones, and E. L. M. Alnowami, "A flexible approach to motion correction in nuclear medicine," IEEE Medical Imaging Conf, (Orlando, Florida, USA), 2009.

[8] M. Alnowani, E. Lewis, M. Guy, and K. Wells, "A marker-less observation model for motion correction in nuclear medicine," SPIE, (San Diego, California, USA), 2010.

[9] S. Anishchenko, V. Osinov, D. Shaposhnikov, L. Podlachikova, R. Comley, and X. Gao, "Toward a robust system to monitor head motions during pet based on facial landmark detection: A new approach," in Computer-Based Medical Systems, 2008. CBMS '08. 21st IEEE International Symposium on, pp. 50-52, June 2008.

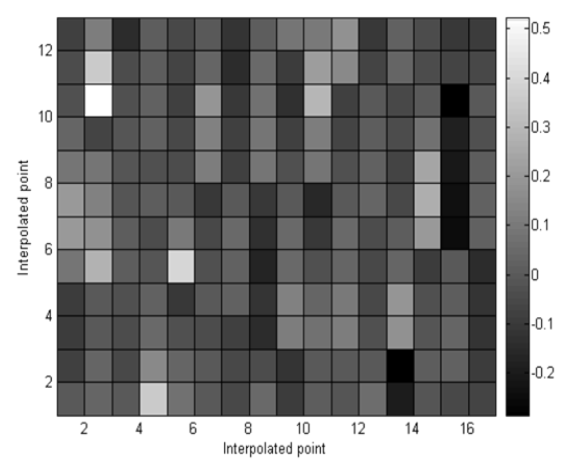

Fig. 5. The error associated with each $13 * 17$ interpolated point in the B-spline model

\section{DisCUSSION AND CONCLUSION}

These preliminary results obtained from the dynamic stereo camera shows that a B-spline model using a uniform control point distribution provides a promising approach to represent the abdominal chest surface without recourse to physical markers. In addition, it will provide a quantitative comparison of the b-spline model with volunteer data obtained at different points in the respiratory cycle and using stomach vs. chest parametric models of respiratory motion. Assessment of the marker less system alongside a conventional marker based system reflects that the average error associated with this model is $0.52 \pm 0.11 \mathrm{~mm}$. Moreover, using the B-spline model represents a saving of more than $90 \%$ over storing the raw frame data form $38 \mathrm{~GB}$ to about $0.4 \mathrm{MB}$ for one tracking session.

\section{REFERENCES}

[1] M. V. Knopp and H. G. Bischoff Radiologe 34(10), pp. 588-91, 1994.

[2] J. Daouk, L. Fin, P. Bailly, and M. E. Meyer Acta Radiol 50(2), pp. 144-55, 2009. 